\title{
Laser-assisted Delivery of a Combined Antioxidant Formulation Enhances the Clinical Efficacy of Fractional Microneedle Radiofrequency Treatment: A Pilot Study
}

\author{
Jihee $\mathrm{Kim}^{1,2,3}$ \\ Soo Min Kim ${ }^{1}$ \\ Bok Ki Jung ${ }^{4}$ \\ Sang Ho Oh \\ Young-Koo Kim ${ }^{5}$ \\ Ju Hee Lee ${ }^{1,2}$
}

\footnotetext{
${ }^{1}$ Department of Dermatology and Cutaneous Biology Research Institute, Severance Hospital, Yonsei University College of Medicine, Seoul, Korea

${ }^{2}$ Scar Laser and Plastic Surgery Center, Yonsei Cancer Hospital, Seoul, Korea

${ }^{3}$ Department of Dermatology, Yongin Severance Hospital, Yongin, Korea

${ }^{4}$ Department of Plastic and Reconstructive Surgery, Yongin Severance Hospital, Yongin, Korea

${ }^{5}$ Yonsei Star Skin and Laser Clinic, Seoul, Korea
}

Received May 25, 2021

Accepted June 7, 2021

\author{
Correspondence \\ Ju Hee Lee \\ Department of Dermatology and Cutaneous \\ Biology Research Institute, Severance Hospital, \\ Yonsei University College of Medicine, 50-1 \\ Yonsei-ro, Seodaemun-gu, Seoul 03722, Korea \\ Tel.: +82-2-2228-2080 \\ Fax: +82-2-393-6947 \\ E-mail: juheedayuhs.ac
}

(c) Korean Society for Laser Medicine and Surgery

(c) This is an open access article distributed under the terms of the Creative Commons Attribution NonCommercial License (http://creativecommons.org/ licenses/by-nc/4.0) which permits unrestricted noncommercial use, distribution, and reproduction in any medium, provided the original work is properly cited.

\begin{abstract}
Background and Objectives
Fractional microneedle radiofrequency systems are popular options to increase elasticity in aging skin. Laser-assisted drug delivery is a promising method for the epidermal injection of topically applied drugs and cosmetic ingredients. This study assesses the safety and efficacy of topical delivery of $L$-ascorbic acid, vitamin $E$, and ferulic acid after fractional microneedle radiofrequency treatment for reducing photodamage.
\end{abstract}

\section{Materials and Methods}

In this prospective, single-center, split-face, controlled pilot study, six women (mean age, 48.0 years; range, 35-57 years; Fitzpatrick skin types III and IV) exhibiting mild to moderate photodamage, underwent a single session of fractional microneedle radiofrequency treatment. The patients were instructed to apply the antioxidant formulation to only one side of the face. Patients were evaluated 3 days, 7 days, and 4 weeks thereafter, using three-dimensional imaging and ultrasound. Ex vivo, the fullthickness human skin was used for molecular and histological evaluation. Statistical analysis was achieved by applying $t$-tests, MannWhitney $U$ tests, and one-way analyses of variance.

\section{Results}

Compared to the untreated side, the antioxidant-treated side exhibited a significant increase in dermal thickness (10.32\% vs. $17.54 \%, p<0.05)$, but not in skin elasticity $(4.76 \%$ vs. $4.69 \%, p>0.05)$. The difference in erythema between the sides was statistically not significant ( $p>0.05)$. In the ex vivo model, expression of FGF2 in the skin was significantly increased after application of the antioxidant formulation, as compared to results obtained subsequent to fractional microneedle radiofrequency treatment only $(p<0.01)$.

\section{Conclusion}

This study demonstrates that for the treatment of photodamaged skin, laser-assisted delivery of the antioxidant formulation is a safe and effective adjuvant modality following fractional microneedle radiofrequency.

\section{Key words}

Energy-based device; Fractional microneedle radiofrequency; Laserassisted drug delivery; Skin aging; Antioxidant 


\section{INTRODUCTION}

Early signs of facial skin aging occur due to changes in tissue elasticity and collagen degradation causing fineto-deep skin folds. ${ }^{1}$ As treatments for facial rejuvenation become more popular, lasers and energy-based devices are increasingly prevalent. ${ }^{2,3}$ There has been evidence to suggest that radiofrequency (RF) devices have a favorable effect on photoaged skin. ${ }^{3}$ High-frequency alternating electrical currents induce neocollagenesis and elastogenesis by volumetric heating of the dermis and subdermal tissue. ${ }^{2}$ Monopolar and bipolar RF-based systems exhibit comparable or even superior efficacy in facial rejuvenation compared to traditional ablative procedures. ${ }^{2}$ Electromagnetic radiation induces collagen contraction and remodeling by leading to the generation of heat shock proteins and matrix metalloproteinases; this contributes to a sustained dermal-remodeling effect. ${ }^{4,5}$ Fractional microneedle RF (FMRF) systems are used to apply microdoses of bipolar RF to the skin to create columns of thermal damage. The microneedle electrode, either insulated or non-insulated, directly penetrates the epidermis for the delivery of electromagnetic pulses, which cause the formation of microthermal zones (MTZs) of denatured collagen. ${ }^{6}$

Laser-assisted drug delivery (LADD) is most commonly performed using ablative fractional lasers (AFLs), and MTZs can improve the passive uptake of delivered molecules by enhancing penetration of the skin. ${ }^{7.8}$ The use of RF systems can enhance skin permeation of macromolecules or hydrophilic agents similar to the microporation generated using ultrasound. ${ }^{9}$ Histologically, fractional RF devices generate more superficial and broader MTZs compared to various AFL devices. ${ }^{10}$

Exposure to UV-irradiation is the primary cause of skin damage; it leads to the production of reactive oxygen species (ROS) and the activation of cell surface receptors, thereby inducing an inflammatory cascade. ${ }^{11}$ Therefore, antioxidants play a substantial role in preventing skin aging due to UV exposure. 12,13 The skin's endogenous and exogenous antioxidants are depleted by repeated UV exposure. ${ }^{14}$ Vitamin C, L-ascorbic acid, is a hydrophilic antioxidant that acts as an ROS scavenger after UV exposure, and it reduces the formation of sunburn cells and erythema. ${ }^{15,16}$ Efficacious percutaneous absorption of $L$ ascorbic acid depends on its formulation and the delivery vehicle when applied topically to the skin. Among various derivatives and isoforms, lipophilic esterified $L$-ascorbic acid has exhibited the highest propensity for percutaneous absorption. ${ }^{17}$ Adding $\alpha$-tocopherol and ferulic acid stabilizes the formulation and enhances photoprotection. ${ }^{15,16}$ Furthermore, $L$-ascorbic acid is involved in stimulating collagen biosynthesis.

To our knowledge, no prospective, randomized, controlled clinical trials have been conducted with regard to FMRF systems. We hypothesized that topical antioxidant supplementation would improve wound healing by providing a scaffold for collagen repair and regrowth. Here, we report the clinical results of a pilot study on facial photoaging after topical application of $L$-ascorbic acid, vitamin $E$, and ferulic acid.

\section{MATERIALS AND METHODS}

\section{Study design and participant selection}

This was a prospective, split-face, single-blind, controlled pilot study conducted at our institution from 1 February 2019 to 31 June 2019. Six female participants Imean age, 48.0 years; range, 35-57 years; Fitzpatrick skin types III and IV) exhibiting mild to moderate photodamage (Glogau scale ${ }^{18} \geq 2$ ) were included in this study, which was approved by the Institutional Review Board of our institution. Subjects were excluded if they were younger than 20 years, older than 60 years, had a history of keloid scarring, were pregnant, had uncontrolled medical illness, or had used any cosmetics containing antioxidant constituents in the 6 months before the treatment. Patients who had received soft-tissue augmentation materials or nonablative laser treatments in the six months before the study were also excluded from the study.

\section{Treatment}

One hour prior to the procedure, topical anesthetics (2.5\% lidocaine $\mathrm{HCl}$ and $2.5 \%$ prilocaine cream) were applied under occlusion. All patients underwent full-face treatment with a pulse-type FMRF device with 25 noninsulated microneedles in a $5 \times 5$ array (Sylfirm ${ }^{\mathrm{TM}}$; Viol Co., Ltd., Seongnam, Koreal. The RF device was set to a frequency of $2 \mathrm{MHz}$ and the depth of penetration was set to 2.0-2.4 mm, which were equivalent of 100-150 joules of energy delivered at $2 \mathrm{MHz}$ per pulse. Eyelids and perioral areas were not treated. Immediately following FMRF treatment, patients were instructed to apply four to five drops of a topical formulation of $L$-ascorbic acid, vitamin E, and ferulic acid (CE Ferulic ${ }^{\circledR}$, SkinCeuticals Inc., New York, USAl to one side of the face. The side of the face to which the formulation would be applied was randomly designated prior to the experiment. Patients were also instructed to wear sunscreen and apply a moisturizer. 


\section{Assessment of clinical efficacy and safety}

All participants were assessed 3 days, 1 week, and 4 weeks after the FMRF treatment. Treatment was completed 8 weeks after the initial visit. All digital photographs were taken using the same camera settings and were digitally processed using identical imaging programs (Nikon D850, Nikon Corporation, Tokyo, Japan). Photoaging was graded blindly by two independent physicians (J.H.L and C.E.Y) on a 5-point global photoaging scale (GPS). For objective assessment of skin rejuvenation, a three-dimensional (3D) imaging system (Morpheus3D Aesthetic Solution, Morpheus Co., Ltd., Yongin, Koreal ${ }^{19}$ was used to measure the length of the midface curvature from the alar groove to the anterior notch of the ear.

To measure structural changes in the dermis and subcutaneous tissue, and to measure the thickness of the dermis, ultrasound imaging was performed with an Ultrascan UC22 device (Courage + Khazaka electronic GmbH, Cologne, Germany). ${ }^{20}$ Skin elasticity was evaluated using the Cutometer Dual MPA 580 device ICourage+Khazaka electronic $\mathrm{GmbH}$ ).

In order to evaluate adverse effects, changes in skin color were measured as the erythema and melanin indices using narrow-band reflectance spectrophotometry (DermaSpectrometer II; Cortex Technology, Hadsund, Denmark) with 568- and 655-nm probes at every visit.

\section{In vitro study \\ Ex vivo skin preparation and tissue handling}

Residual human skin specimens of women aged 3555 years were acquired from the Department of Plastic Surgery at our institution, which was approved by the Institutional Review Board of our institution. Tissues were washed three times with Dulbecco's phosphate-buffered saline. For UV irradiation, each sample of ex vivo skin was irradiated at $4 \mathrm{~J} / \mathrm{cm}^{2}$ with 8-W UV-A lamps (F8T5BL, Sankyo Denki Co., Ltd., Kanagawa, Japan). The full thickness of the skin specimen was treated with pulse-type FMRF using the same parameters as in the patients (2 $\mathrm{MHz}, 2.4-\mathrm{mm}$ penetration). Thereafter, the specimens were treated with $0.001 \%$ of $L$-ascorbic acid, vitamin $E$, and ferulic acid. Tissues were cultured on a $2 \%$ agarose gel lagarose:Dulbecco's Modified Eagle's Medium = 1:5; Young Sciences, Bucheon, South Koreal in an incubator at $37^{\circ} \mathrm{C}$, under high humidity and $5 \% \mathrm{CO} 2$.

\section{Quantitative real-time PCR (qPCR)}

To investigate the potential mechanism involved in the observed effect of vitamin C, E, and ferulic acid after FMRF treatment, we investigated the effect of gene expression on extracellular matrix (ECM) remodeling in ex vivo human skin. The mRNA expression levels of transforming growth factor-beta (TGF- $\beta$ ), type III collagen (COL3A1), and fibroblast growth factor 2 (FGF2) were analyzed, with glyceraldehyde 3-phosphate dehydrogenase (GAPDH) as reference gene. FMRF-treated skin specimens were collected 3 and 7 days after treatment, for RNA extraction. Total RNA was extracted using an RNeasy Plus Mini Kit (Qiagen, Hilden, Germany) and reverse transcribed to cDNA using an RNA to cDNA EcoDry Premix kit (Takara Bio USA, Inc., Mountain View, California, USA). qPCR was conducted with the TaqMan Gene Expression Master Mix (Applied Biosystems, Foster City, California, USA). The TaqMan Gene Expression assays (Thermo Fisher Scientific, Fisher Scientific Inc., Waltham, Massachusetts, USA) used in this study were as follows: Hs00943809_m1 (COL3A1), Hs00171257_m1 (TGFB1), Hs002666454_m1 (FGF2), and Hs02758991_g1 (GAPDH).

\section{Histological analysis}

Skin samples were biopsied and fixed in a formalin solution (10\%) for $24 \mathrm{~h}$. Paraffin blocks were cut into 4.5- $\mathrm{\mu m}$ thick sections. Sectioned samples were deparaffinized using a xylene and ethanol series at room temperature, and then washed with deionized water. The sectioned samples were stained with hematoxylin and eosin, as well as Masson's trichrome stain to analyze collagen denaturation and deposition.

\section{Immunofluorescence}

To evaluate potential irritation caused by a lowered $\mathrm{pH}$ following application of the antioxidant formulation, we studied the expression of molecules related to the functioning of the skin barrier using immunofluorescence. ${ }^{21}$ As primary antibodies, anti-Filaggrin lab81468, Abcam plc., UK; 1:100), anti-Involucrin (ab53112, Abcam; 1:100), and anti-Loricrin (ab85679, Abcam; 1:100) antibodies were incubated for $24 \mathrm{~h}$ at room temperature. Goat anti-Rabbit $\lg G(H+L)$ Alexa Fluor 555 conjugate (A-21428, Thermo Fisher Scientific; 1:500) and Goat Anti-Rabbit IgG H\&L (FITC) (ab6717, Abcam; 1:2000) were used as secondary antibodies. Samples were rinsed with tris-buffered saline-Tween 20 and mounted with Vectashield containing DAPI (Vector Laboratories, Inc., Burlingame, California, USA). The samples were monitored with a laser-scanning microscope (LSM 700; Carl Zeiss AG, Oberkochen, Germanyl and analyzed with LSM 5 Image Browser software (Carl Zeiss AG). 


\section{Statistical analysis}

The data was tested for statistical significance using a t-test, Mann-Whitney $U$ test, or one-way analysis of variance. The results of the study were statistically significant if $p<0.05$. All data points from the in vitro study are indicated as means and standard deviations, and statistical differences between groups were determined using an unpaired $t$-test. $p$-values $<0.001\left({ }^{* * *}\right),<0.01\left(^{(* *)}\right.$ and $<$ $\left.0.05{ }^{*}\right)$ were considered statistically significant in this study. All statistical analyses were performed using IBM SPSS Statistics for Windows version 23.0 (IBM Corp., Armonk, New York, USA).

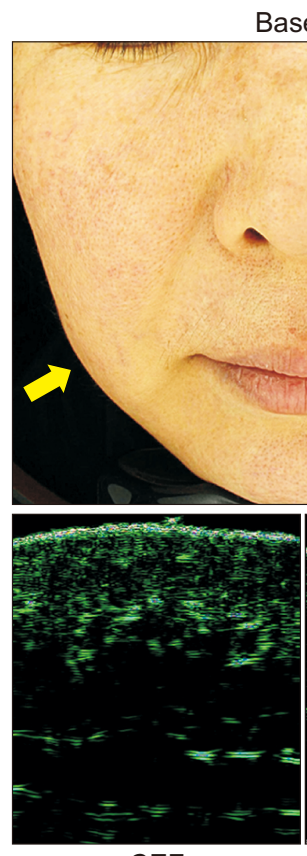

CEF
Baseline
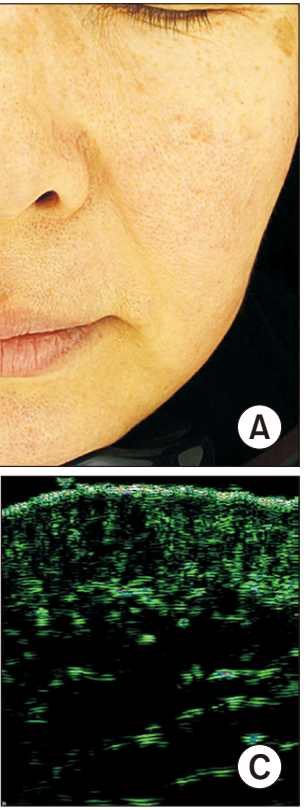

Control

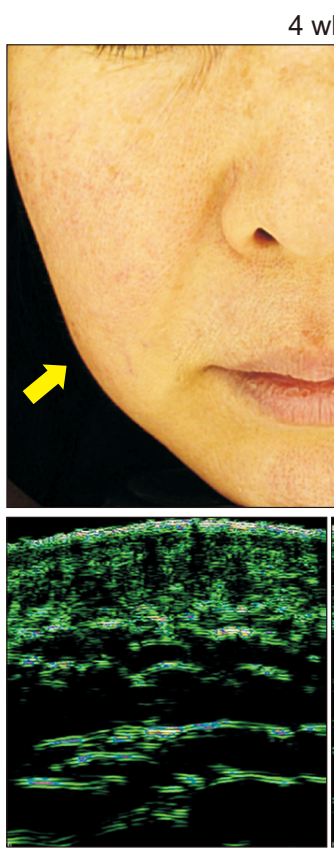

CEF
4 wk

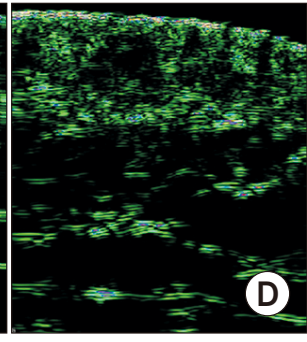

Control
Fig. 1. Clinical images and ultrasound analysis. (A) Digital photograph of patient's skin at baseline and (B) four weeks after a single session of fractional microneedle radiofrequency treatment and daily application of the antioxidant formulation. (C, D) Ultrasound analysis revealed increased echogenicity in the dermal level on the side where the antioxidant formulation was applied.
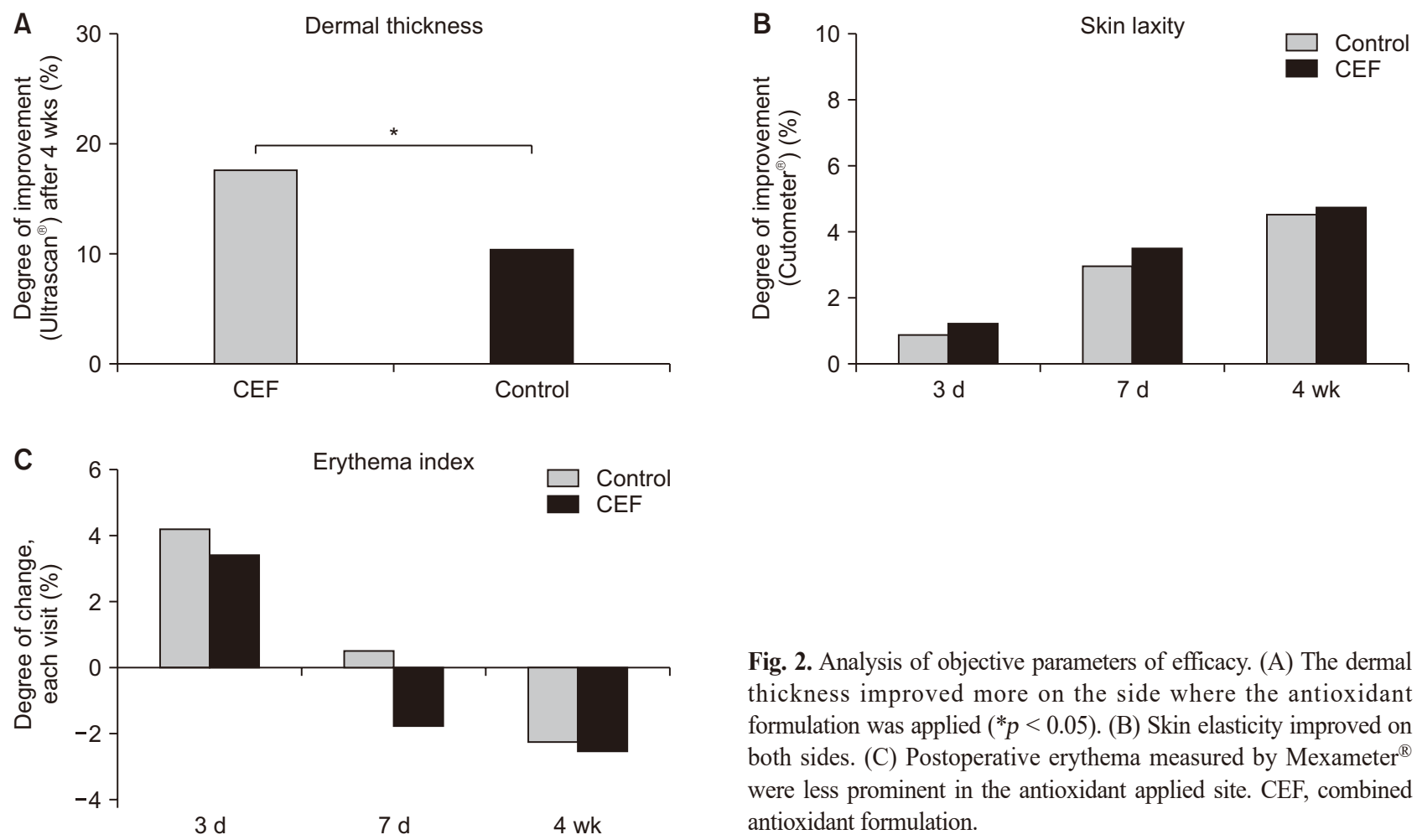

Fig. 2. Analysis of objective parameters of efficacy. (A) The dermal thickness improved more on the side where the antioxidant formulation was applied $\left({ }^{*} p<0.05\right)$. (B) Skin elasticity improved on both sides. (C) Postoperative erythema measured by Mexameter ${ }^{\mathbb{R}}$ were less prominent in the antioxidant applied site. CEF, combined antioxidant formulation. 


\section{RESULTS}

\section{Clinical efficacy}

The mean GPS score was $3.16 \pm 0.73$ at baseline, which decreased to $2.66 \pm 0.52$ on the side where antioxidants were applied and $3.00 \pm 0.58$ on the control side $(p<0.05)$ (Fig. 1A, B).

The increase in average midface curvature length after four weeks was higher on the antioxidant-treated side (from $117.42 \pm 4.58$ to $119.90 \pm 4.64 \mathrm{~mm}$ ) than that on the non-treated side (from $117.23 \pm 4.55$ to $118.53 \pm 4.47 \mathrm{~mm}$; $p<0.05)$. The increase in mean dermal thickness after four weeks, measured using ultrasound, was higher on the treated side lfrom $10.77 \pm 3.80$ to $12.08 \pm 3.59 \mathrm{~mm}$; $17.54 \%$ improvement) than that of the non-treated side (from $9.98 \pm 3.39$ to $10.83 \pm 3.04 ; 10.32 \%$ improvement; $p$ $<0.05$ ) (Fig. 1C, D, and 2A). Skin elasticity increased after four weeks in the treated and control sides, with no statistically significant difference between them ( $p>0.05)$; from
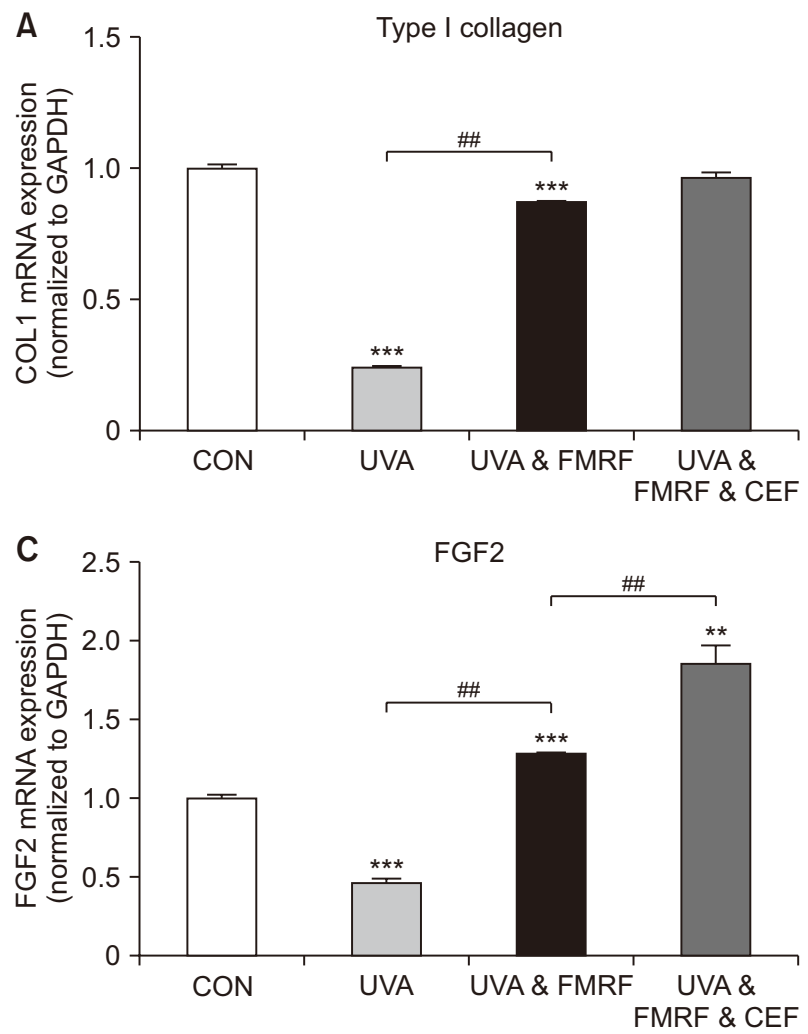

$0.64 \pm 0.07$ to $0.67 \pm 0.72$ and from $0.63 \pm 0.07$ to $0.66 \pm 0.08$ respectively (Fig. 2B).

\section{Safety profile}

After four weeks, no adverse effects were encountered and there were no cases of patient dropout due to side effects. Both the antioxidant-treated and control sides exhibited a decrease in the melanin index after four weeks (from $155.50 \pm 20.53$ to $150.28 \pm 19.63$ and from $158.44 \pm$ 20.83 to $155.89 \pm 23.04$, respectively; $p>0.05$ ). Both sides exhibited a reduction in the erythema index after four weeks, but there was no statistically significant difference between the sides (from $341.94 \pm 63.10$ to $332.67 \pm 60.01$ and from $354.00 \pm 62.70$ to $344.78 \pm 60.21$, respectively; $p$ $>0.05$ ) (Fig. 2C).

\section{In vitro analysis on the laser-assisted delivery of the antioxidant formulation \\ UV-A exposure induced downregulation of TGF- $B$,}

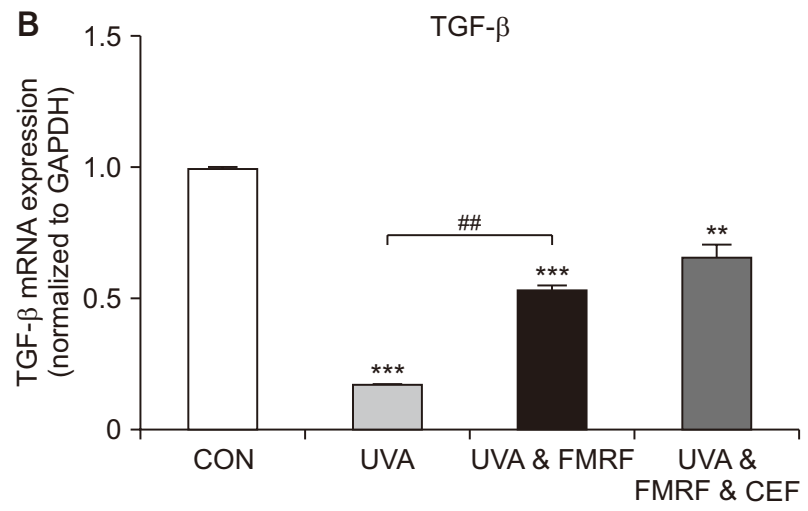

Fig. 3. mRNA transcription of extracellular matrix-related molecules were analyzed in photoaged, ex vivo human skin after fractional microneedle radiofrequency (FMRF) treatment and application of the antioxidant formulation. (A) Expression of type III collagen (COL3A1) was statistically significantly increased after a single session of FMRF treatment. (B) FMRF treatment induced a statistically significant increase in transforming growth factor-beta (TGF- $\beta$ ) expression. (C) Fibroblast growth factor (FGF2) expression increased upon FMRF treatment and increased further upon application of the antioxidant formulation. Data are indicated as mean \pm standard error. Differences between groups were compared using an unpaired t-test. ${ }^{\#}$ indicates a significant difference $(p<0.01)$ between two groups; $*(p<0.05)$ and $* *(p<0.01)$ indicate significant differences from the positive control. CEF, combination antioxidant formulation; COL3A1, type III collagen; FGF2, fibroblast growth factor 2; FMRF, fractional microneedle radiofrequency; TGF- $\beta$, transforming growth factor-beta. 
COL3A1, and FGF2 by 6.25-, 4.35-, and 2.13-fold, respectively (Fig. 3). After FMRF treatment, TGF- $\beta$ and COL3A1 were significantly upregulated compared to the UV-Aonly group, and FGF2 levels were even increased by 1.29 fold compared to positive controls. Additionally, treatment with the antioxidant formulation resulted in a further increase in FGF2 transcription, to 1.86-fold that of positive controls (Fig. 3C).

Following UV-A exposure, FMRF generated MTZs, with collagen denaturation reaching down to the dermis (Fig. 4). The explanted skin exhibited increased COL3A1 expression, detected using Masson's trichrome stain, after a single pulse-type FMRF treatment (Fig. 5A, B); this expression was further increased after treatment with the antioxidant formulation (Fig. 5C).

Surprisingly, application of the antioxidant formulation did not affect expression levels of filaggrin (Fig. 6A) and loricrin (Fig. 6B) on the stratum corneum or throughout the epidermal layers.

\section{DISCUSSION}

We assessed the efficacy and safety of the application of an antioxidant formulation after FMRF treatment among patients with photoaging. The clinical signs of photorejuvenation, such as midface volume and dermal thickness, improved more on the treated side than on the untreated side of the face. No patients experienced adverse skin reactions after the procedure. In addition, the immediate application of the antioxidant formulation after laser treatment prevents post-treatment downtime.

Radiofrequency devices are known to evoke a plasma spark when used to irradiate skin. ${ }^{6,10}$ In previous studies, the depth and width of the microchannel induced using a fractional CO2 laser in an ex vivo pig skin model increased with the energy level used. ${ }^{710}$ Previous stud-

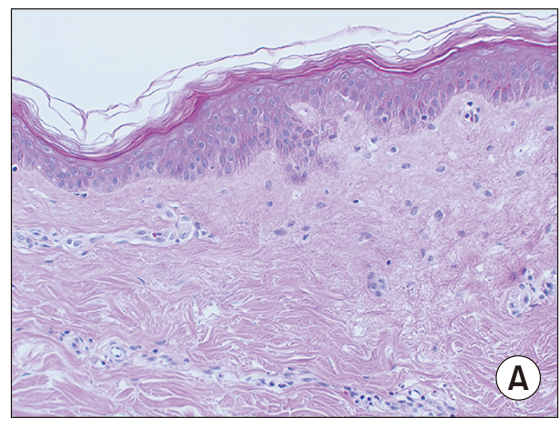

Control

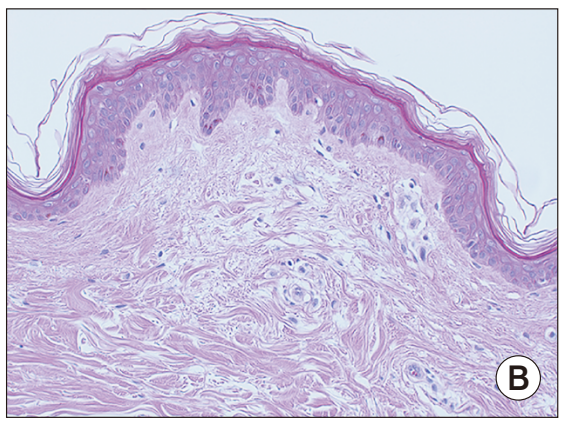

UVA

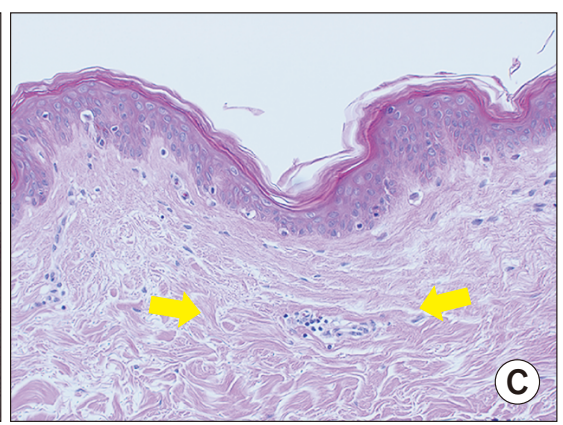

FMRF after UVA

Fig. 4. Histological analysis of photoaged ex vivo human skin after fractional microneedle radiofrequency (FMRF) treatment. (A, B) H\&E staining of UV-A irradiated skin revealed solar elastosis and hyalinization of collagen on the upper dermis $(\times 100)$. (C) A single pulse-type FMRF treatment induced microthermal zones (indicated by yellow arrows), which induced the generation of thicker collagen bundles $(\mathrm{H} \& \mathrm{E}, \times 100)$. FMRF, fractional microneedle radiofrequency; $\mathrm{H} \& \mathrm{E}$, hematoxylin-eosin.

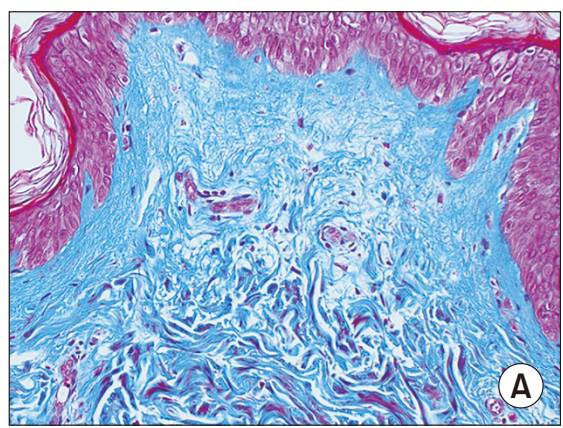

UVA

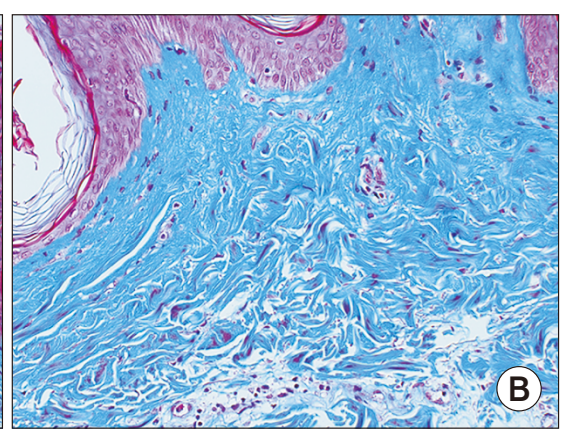

FMRF \& UVA

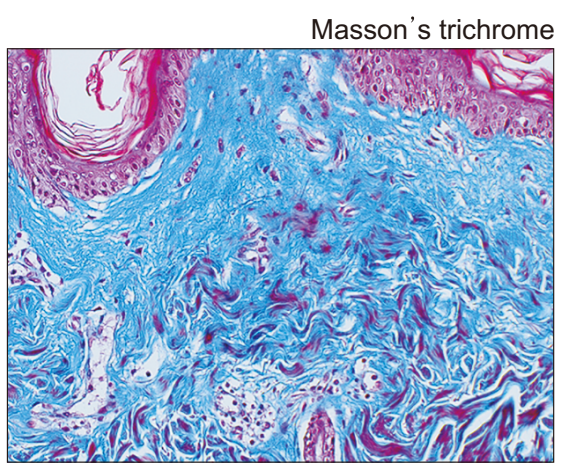

CEF after FMRF \& UVA

Fig. 5. Histological analysis of photoaged ex vivo human skin after fractional microneedle radiofrequency (FMRF) and application of an antioxidant formulation. (A, B) A single pulse-type FMRF treatment induced neocollagenesis on the photoaged skin (Masson's trichrome, $\times 100$ ). (C) Subsequent treatment with the antioxidant formulation induced a further increase in collagen deposition in the FMRF-treated skin (Masson's trichrome, $\times 100)$. FMRF, fractional microneedle radiofrequency. 


\section{Fllaggrin}
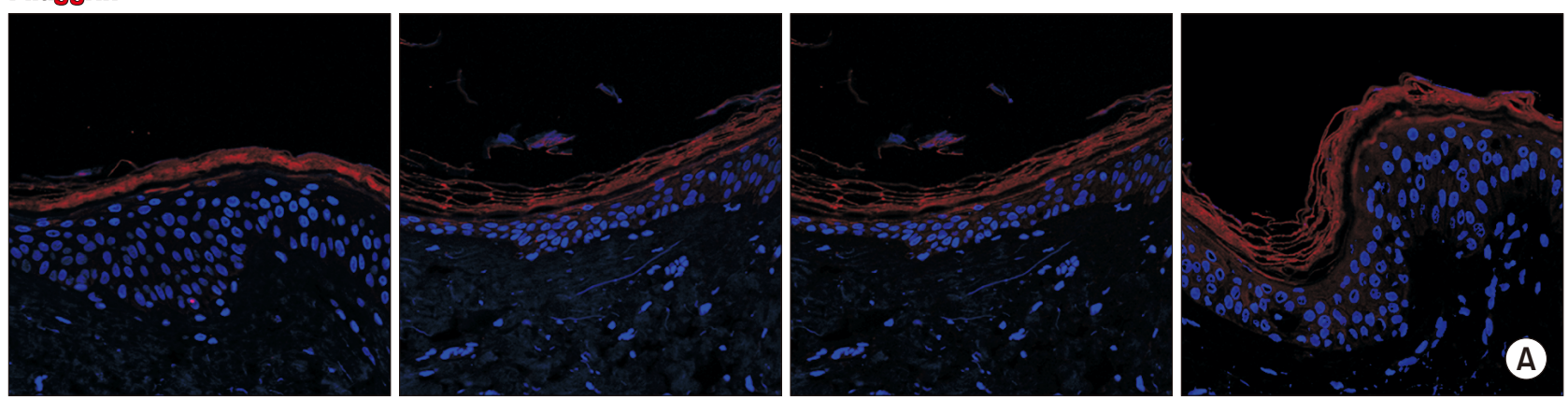

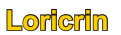

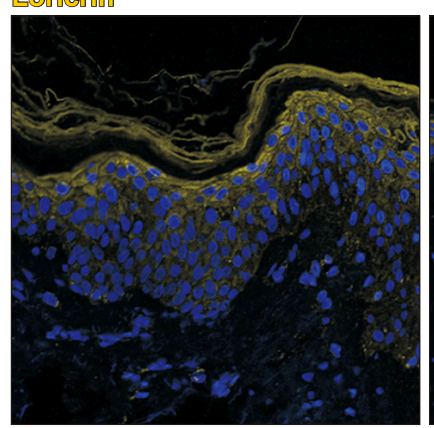

Control

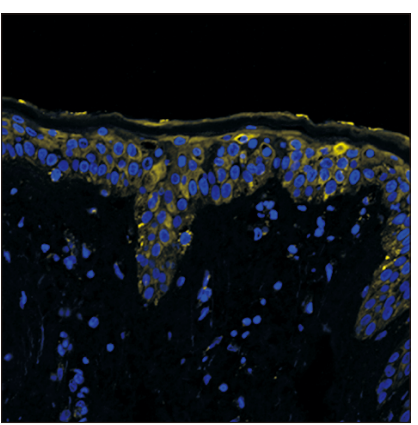

UVA

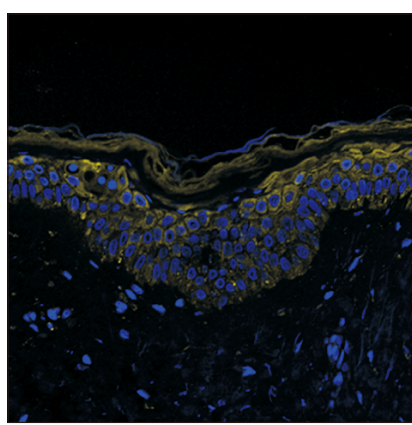

FMRF \& UVA

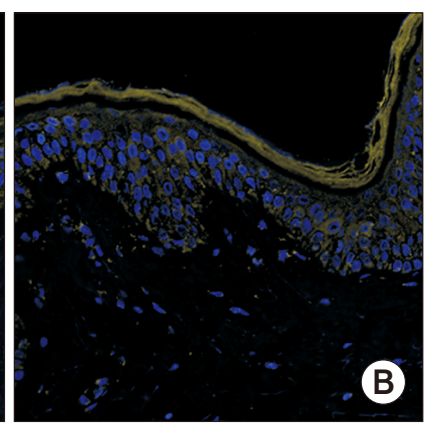

CEF after FMRF \& UVA

Fig. 6. Expression of skin barrier molecules was analyzed using immunofluorescence. (A) Expression of filaggrin was unaffected by topical application of the antioxidant formulation after fractional microneedle radiofrequency treatment $(\times 200)$. (B) Expression of loricrin was similarly unaffected $(\times 200)$.

ies on RF treatment revealed that the energy generated by each column in the MTZ exhibits minimal spread to the surrounding tissues, thus ensuring the stability of the epidermis between each MTZ. To target photoaged skin, deeply penetrating microablative zones are required as a conduit for active delivery of molecules to induce a sufficient clinical effect. ${ }^{22}$ FMRF treatment has been suggest as an efficient alternative for LADD to ablative fractional laser systems, as it can be used to generate microporation with its microneedles. ${ }^{23}$

We used an ex vivo model of photoaging to assess potential molecular and histological changes after FMRF and LADD of an antioxidant formulation. Generally, FMRF-induced neocollagenesis is expected to occur 1 to 3 months after a single treatment. ${ }^{24,25}$ The thermal response of skin to treatment with FMRF induces a tissuerepair cascade leading to long-term dermal remodeling with increased collagen synthesis. ${ }^{24}$

In general, procedures for photorejuvenation commonly cause substantial patient discomfort, a prolonged downtime, and adverse events such as postprocedural erythema and hyperpigmentation. ${ }^{26,27}$ However, incorporation of topical antioxidants has been demonstrated to facilitate the tissue-repair process and minimize post- treatment downtime. ${ }^{8}$ In our pilot study, we demonstrated that posttreatment application of an antioxidant formulation can increase the clinical efficacy of FMRF treatment and reduce posttreatment erythema. Additionally, we have demonstrated that the application of the low-pH antioxidant formulation did disrupt skin barrier-function, as the expression of filaggrin and loricrin in the stratum corneum was unaffected in an ex vivo model of photodamaged skin. Moreover, our histological data suggest that combining FMRF treatment with LADD of a formulation containing $L$-ascorbic acid, vitamin $E$, and ferulic acid leads to increased collagen synthesis.

The main limitation of our study is in its small sample size; therefore, the reliability of the statistical analyses needs to be verified in larger-scale studies. The follow-up period of this study was only four weeks; therefore, longterm assessments are needed to clarify the potential effects of the treatment on neocollagenesis. In this pilot study, we have provided preliminary evidence that agrees with our hypothesis that topical antioxidant supplementation improves wound healing by providing a scaffold for collagen repair and regrowth. We believe that further research will increase the use of multimodal combination treatments, exploiting a synergistic effect between FMRF 
treatment and LADD of different antioxidant formulations, improving skin treatment in general.

In conclusion, we discovered that use of a combined antioxidant formulation increased dermal thickness after FMRF treatment, and decreased postprocedural erythema and patient downtime. In addition, we demonstrated the sustained effect of laser-assisted delivery of the antioxidant formulation on ECM remodeling in vitro. Further clinical studies with a larger population and extended follow-up period are warranted to support the combined use of FMRF treatment and antioxidant application in skin renewal.

\section{CONFLICT OF INTEREST}

The antioxidant formulation (CE Ferulic ${ }^{\circledR}$, SkinCeuticals Inc., New York, USA) used in the study was provided by SkinCeuticals. However, SkinCeuticals had no role in the study design, implementation, data collection, data analysis, data interpretation, manuscript preparation, manuscript review, or manuscript approval. There are no other conflicts of interest to declare.

\section{FUNDING}

This study was supported by the Korea Medical Device Development Fund grant funded by the Korea government (the Ministry of Science and ICT, the Ministry of Trade, Industry and Evergy, the Ministry of Health \& Welfare, Republic of Korea, the Ministry of Food and Drug Safety) (Project Number: 202014X4502). This work was also supported by the grant of the Korea Mental Health Technology R\&D Project, Ministry of Health \& Welfare, Republic of Korea (2020-31-0659) and Yonsei University Faculty Research (Grant 6-2020-0081).

\section{REFERENCES}

1. Nkengne A, Bertin C. Aging and facial changes--documenting clinical signs, part 1: clinical changes of the aging face. Skinmed 2013;11:281-6.

2. Britt CJ, Marcus B. Energy-based facial rejuvenation: advances in diagnosis and treatment. JAMA Facial Plast Surg 2017;19:6471.

3. Gold MH. The future of non-invasive rejuvenation technology: devices. J Drugs Dermatol 2017;16:s104-7.

4. Hantash BM, Ubeid AA, Chang H, Kafi R, Renton B. Bipolar fractional radiofrequency treatment induces neoelastogenesis and neocollagenesis. Lasers Surg Med 2009;41:1-9.

5. Manuskiatti W, Pattanaprichakul $P$, Inthasotti S, Sitthinamsu- wan P, Hanamornroongruang S, Wanitphakdeedecha R, et al. Thermal response of in vivo human skin to fractional radiofrequency microneedle device. Biomed Res Int 2016;2016:6939018.

6. Manstein D, Herron GS, Sink RK, Tanner H, Anderson RR. Fractional photothermolysis: a new concept for cutaneous remodeling using microscopic patterns of thermal injury. Lasers Surg Med 2004;34:426-38.

7. Waibel JS, Mi QS, Ozog D, Qu L, Zhou L, Rudnick A, et al. Laserassisted delivery of vitamin $C$, vitamin $E$, and ferulic acid formula serum decreases fractional laser postoperative recovery by increased beta fibroblast growth factor expression. Lasers Surg Med 2016;48:238-44.

8. Levin G, Gershonowitz A, Sacks H, Stern M, Sherman A, Rudaev $S$, et al. Transdermal delivery of human growth hormone through RF-microchannels. Pharm Res 2005;22:550-5.

9. Shin MK, Choi JH, Ahn SB, Lee MH. Histologic comparison of microscopic treatment zones induced by fractional lasers and radiofrequency. J Cosmet Laser Ther 2014;16:317-23.

10. Scharffetter-Kochanek K, Wlaschek M, Brenneisen P, Schauen M, Blaudschun R, Wenk J. UV-induced reactive oxygen species in photocarcinogenesis and photoaging. Biol Chem 1997;378:1247-57.

11. Naylor EC, Watson RE, Sherratt MJ. Molecular aspects of skin ageing. Maturitas 2011;69:249-56.

12. Kohl E, Steinbauer J, Landthaler M, Szeimies RM. Skin ageing. J Eur Acad Dermatol Venereol 2011;25:873-84.

13. Rhie G, Shin MH, Seo JY, Choi WW, Cho KH, Kim KH, et al. Aging- and photoaging-dependent changes of enzymic and nonenzymic antioxidants in the epidermis and dermis of human skin in vivo. J Invest Dermatol 2001;117:1212-7.

14. Lin FH, Lin JY, Gupta RD, Tournas JA, Burch JA, Selim MA, et al. Ferulic acid stabilizes a solution of vitamins $C$ and $E$ and doubles its photoprotection of skin. J Invest Dermatol 2005; 125:826-32.

15. Lin JY, Selim MA, Shea CR, Grichnik JM, Omar MM, MonteiroRiviere NA, et al. UV photoprotection by combination topical antioxidants vitamin C and vitamin E. J Am Acad Dermatol 2003;48:866-74.

16. Pinnell SR, Yang H, Omar M, Monteiro-Riviere N, DeBuys HV, Walker LC, et al. Topical L-ascorbic acid: percutaneous absorption studies. Dermatol Surg 2001;27:137-42.

17. Alexiades-Armenakas M. A quantitative and comprehensive grading scale for rhytides, laxity, and photoaging. J Drugs Dermatol 2006;5:808-9.

18. Rho NK, Park JY, Youn CS, Lee SK, Kim HS. Early changes in facial profile following structured filler rhinoplasty: an anthropometric analysis using a 3-dimensional imaging system. Dermatol Surg 2017;43:255-63.

19. Dąbrowska M, Mielcarek A, Nowak I. Evaluation of sex-related changes in skin topography and structure using innovative skin 
testing equipment. Skin Res Technol 2018;24:614-20.

20. Thyssen JP, Kezic S. Causes of epidermal filaggrin reduction and their role in the pathogenesis of atopic dermatitis. J Allergy Clin Immunol 2014;134:792-9.

21. Ibrahim O, Wenande E, Hogan S, Arndt KA, Haedersdal M, Dover JS. Challenges to laser-assisted drug delivery: applying theory to clinical practice. Lasers Surg Med 2018;50:20-7.

22. Hansen FS, Wenande E, Haedersdal M, Fuchs CSK. Microneedle fractional radiofrequency-induced micropores evaluated by in vivo reflectance confocal microscopy, optical coherence tomography, and histology. Skin Res Technol 2019;25:482-8.

23. Kaminaka C, Uede M, Nakamura Y, Furukawa F, Yamamoto Y. Histological studies of facial acne and atrophic acne scars treated with a bipolar fractional radiofrequency system. J Dermatol 2014:41:435-8.

24. Pudukadan D. Treatment of acne scars on darker skin types using a noninsulated smooth motion, electronically controlled radiofrequency microneedles treatment system. Dermatol Surg 2017;43 Suppl 1:S64-9.
25. Metelitsa Al, Alster TS. Fractionated laser skin resurfacing treatment complications: a review. Dermatol Surg 2010;36:299306.

26. Chan HH, Manstein D, Yu CS, Shek S, Kono T, Wei WI. The prevalence and risk factors of post-inflammatory hyperpigmentation after fractional resurfacing in Asians. Lasers Surg Med 2007;39:381-5.

27. Waibel J, Mi QS. Split face comparison to evaluate synergistic response of fractional ablative laser and vitamin $\mathrm{C}$, vitamin $\mathrm{E}$, and ferulic acid on molecular wound healing factors and to clinically decrease postoperative wound healing time. J Am Acad Dermatol 2014;70(5 Suppl 1):AB204.

How to cite this article: Kim J, Kim SM, Jung BK, Oh SH, Kim YK, Lee JH. Laser-assisted delivery of a combined antioxidant formulation enhances the clinical efficacy of fractional microneedle radiofrequency treatment: a pilot study. MedLasers2021;10:161-169.https://doi.org/10.25289/ML.2021.10.3.161 